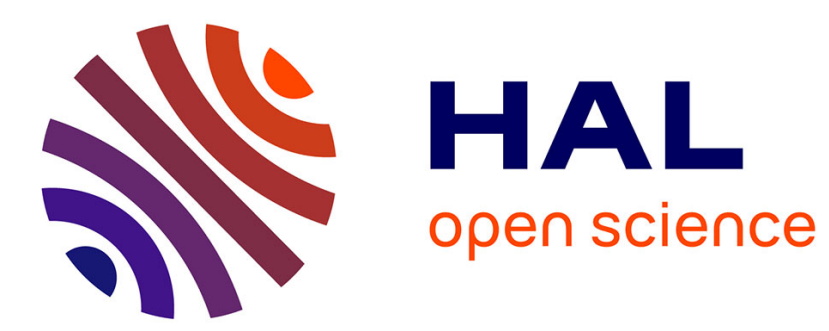

\title{
Alignment dynamics of diffusive scalar gradient in a two-dimensional model flow
}

\author{
Michel Gonzalez
}

\section{To cite this version:}

Michel Gonzalez. Alignment dynamics of diffusive scalar gradient in a two-dimensional model flow. Theoretical and Computational Fluid Dynamics, 2018, 32 (4), pp.521-533. 10.1007/s00162-018-0459z . hal-02123049

\section{HAL Id: hal-02123049 \\ https://hal.science/hal-02123049}

Submitted on 9 May 2019

HAL is a multi-disciplinary open access archive for the deposit and dissemination of scientific research documents, whether they are published or not. The documents may come from teaching and research institutions in France or abroad, or from public or private research centers.
L'archive ouverte pluridisciplinaire HAL, est destinée au dépôt et à la diffusion de documents scientifiques de niveau recherche, publiés ou non, émanant des établissements d'enseignement et de recherche français ou étrangers, des laboratoires publics ou privés. 


\title{
Alignment dynamics of diffusive scalar gradient in a two-dimensional model flow
}

\author{
M. Gonzalez
}

Received: date / Accepted: date

\begin{abstract}
The Lagrangian two-dimensional approach of scalar gradient kinematics is revisited accounting for molecular diffusion. Numerical simulations are performed in an analytic, parameterized model flow, which enables considering different regimes of scalar gradient dynamics. Attention is especially focused on the influence of molecular diffusion on Lagrangian statistical orientations and on the dynamics of scalar gradient alignment.
\end{abstract}

Keywords Scalar gradient - Molecular diffusion - Lagrangian statistics · Alignment dynamics

\section{Introduction}

A number of basic and practical questions in fluid dynamics are connected to the transport of vectors $[1,2]$, an issue therefore relevant to many fields - process engineering, reacting flows, mixing, astrophysical flows, etc. Vectors defining material lines or surfaces, vorticity, the vorticity gradient in two-dimensional flows, gradients of scalar quantities such as concentration or temperature, the magnetic field vector in magnetohydrodynamics, are transported vectors.

Most often, analyses are with regard the growth - or decay - of the magnitude of the transported vector. The growth of the scalar gradient, for example, indicates the production of small scales in the scalar field; addressing the dynamo effect needs to determine the physical conditions in which the magnetic field vector is amplified. The bare kinematics of vector amplification - in non-diffusive tracer advection, kinematic dynamo, or inviscid vortex stretching - is a matter of strain level and orientation within the strain eigenframe. Just like strain intensity, vector orientation is essential to the process and was investigated in many studies [3-7] (see [2] for more references).

M. Gonzalez

CNRS, UMR 6614/CORIA, Site universitaire du Madrillet

76801 Saint-Etienne du Rouvray, France

E-mail: michel.gonzalez@coria.fr 
A general approach of vector kinematics is especially difficult when an active vector such as vorticity, the magnetic field in the dynamic regime, or the gradient of a non-passive scalar is considered $[6,8,9]$. Lapeyre et al. [5] showed that the analytic approach is tractable for a passive, non-diffusive vector - the gradient of a tracer in their specific case - transported by a two-dimensional flow, provided that an adiabatic hypothesis is made (Lagrangian variations of strain persistence are slow enough for the vector to respond to the velocity gradient tensor). An extension to three-dimensional flows was proposed [10,11], and the relaxation of the adiabatic hypothesis was examined $[12,13]$.

By breaking the universal nature of the inviscid vector kinematics strictly determined by the velocity gradient tensor, molecular diffusion makes general analyses much harder. However, viscous and diffusive effects deserve special attention. The purely inertial view indeed does not account for the full process, for there is a range of scales where the mechanics of vectors is itself affected by viscous and diffusive effects. This point is especially crucial in the study of cascade and small-scale phenomena. Modelling vector and tensor fields [14,15,11] also needs addressing the influence of molecular processes.

Some previous works specifically addressed the effects of viscosity or molecular diffusion on vector alignment. In particular, viscosity was shown to influence vorticity alignment [16] and vortex lines geometry [17,18]. In two-dimensional turbulence, Lapeyre et al. [19] analyzed the role of molecular diffusion on the orientation dynamics of scalar gradient and vorticity gradient, and Protas et al. [20] studied the Reynolds-number dependence of the vorticity gradient alignment. In three-dimensional turbulence, the effects of both the Reynolds and Schmidt numbers on the alignment of the scalar gradient were examined by Vedula et al. [21]. Brandenburg et al. [22] addressed the influence of the magnetic diffusivity on the alignment of flux lines of the magnetic field. Recently, a Eulerian numerical study [23] confirmed the basic analysis of Constantin et al. [18] regarding the alignment and structure of a diffusive vector field.

The present study is based on a Lagrangian approach. The latter enables the simulation of the kinematics of a scalar gradient at infinite Péclet number which is considered as the reference case. Accounting for molecular diffusion in the Lagrangian procedure is performed by simultaneously solving the Eulerian equations for the scalar gradient components. The influence of molecular diffusion upon alignment properties is checked for decreasing Péclet number. The analysis is specifically focused on the way in which the inviscid alignment and dynamics of scalar gradient orientation are altered as the Péclet number is decreased. The analytic, two-dimensional, model flowfield was already shown to include the essential features of flow structure for investigating the kinematics of transported vectors [23]. By simply changing the value of a flow parameter, different regimes are considered as regards the degree of adiabaticity of scalar gradient dynamics. In this respect, the analysis is more general than the previous, Eulerian one [23].

The equations for the gradient of a scalar are given in Section 2. The model flowfield is defined in Section 3. Section 4 is devoted to the description of the numerical method. The numerical results are discussed in Section 5, and conclusions are drawn in Section 6 . 


\section{Equations for the gradient of a scalar}

In an incompressible flow, the Lagrangian equation for the gradient, $G=\nabla \Theta$, of a scalar $\Theta$ is:

$$
\frac{D \mathrm{G}}{D t}=-\mathrm{A}^{T} \cdot \mathrm{G}+D \Delta \mathrm{G}
$$

where $\mathrm{A}$ is the velocity gradient tensor, and $D$ is the diffusion coefficient of the scalar.

With $\theta$ the orientation of $\mathrm{G}$ in the fixed frame of reference, the equations for the norm and the orientation of vector $\mathrm{G}=G(\cos \theta, \sin \theta)$ are [5]:

$$
\begin{gathered}
\frac{D G}{D t}=-\frac{\sigma}{2} \sin [2(\theta+\Phi)] G+D \Delta G-D|\nabla \theta|^{2} G, \\
\frac{D \theta}{D t}=\frac{1}{2}\{\omega-\sigma \cos [2(\theta+\Phi)]\}+D \Delta \theta+2 D \frac{\nabla G \cdot \nabla \theta}{G},
\end{gathered}
$$

where $\sigma=\left(\sigma_{n}^{2}+\sigma_{s}^{2}\right)^{1 / 2}$ is the strain intensity, with $\sigma_{n}=\partial u / \partial x-\partial v / \partial y$ and $\sigma_{s}=\partial u / \partial y+\partial v / \partial x$, the normal and shear strain components, respectively; $\omega=$ $\partial v / \partial x-\partial u / \partial y$ is the vorticity and $\Phi$, given by $\tan (2 \Phi)=\sigma_{n} / \sigma_{s}$, is the orientation of the strain principal axes in the fixed frame of reference, $(x, y)$.

Special alignments of the scalar gradient are $\theta_{d}=-\Phi+\pi / 4$ (extensional strain direction), for which the norm growth rate reaches its minimum value, $-\sigma / 2$, and $\theta_{c}=-\Phi-\pi / 4$ (compressional strain direction), for which it takes the maximum value, $\sigma / 2$. At infinite Péclet number, an additional special alignment, the equilibrium direction, can be defined when strain prevails (Section 5.1.1).

Equations (2) and (3) show the effects of the flow and of molecular diffusion on the vector norm and orientation. The action of the flow consists of the straining of the vector norm - first term on the right-hand side of Eq. (2) -, and changes in angle $\theta$ due to strain and rotation - first term on the right-hand side of Eq. (3).

Diffusive terms split up into linear, Laplacian terms, and non-linear terms. The former, $D \Delta G$ and $D \Delta \theta$, express diffusive smoothing; the latter, $-D|\nabla \theta|^{2} G=$ $\mathcal{D}_{n l}(G)$ and $2 D(\nabla G . \nabla \theta) / G=\mathcal{D}_{n l}(\theta)$, express dissipation caused by angle gradients, and diffusive tilting resulting from the interaction between norm gradient and orientation gradient. Detailed analyses of these terms were made in previous studies $[18,23]$.

\section{Model flow}

As was done in a previous, Eulerian study [23], the flow is specialized to a twocomponent velocity field, namely $(u, v, w)=(\partial \psi / \partial y,-\partial \psi / \partial x, 0)$, with the general form of the streamfunction, $\psi$, given by:

$$
\psi=\alpha\{\cos [y+\cos (t)]+\sin [x+\sin (t)]\}+\delta[\cos (y)-\sin (x)],
$$

where $\alpha=\sqrt{3 / 2-\delta^{2}}$. This family of flows was defined by Tanner and Hughes [24] by combining the Galloway-Proctor, circularly polarized, unsteady flow [25], with a steady, two-dimensional, cat's-eye-type flow [26]. Making $\delta$ spanning the range $[0, \sqrt{3 / 2}]$ continuously shifts the flow characteristics from those of the unsteady flow to those of the steady flow. 
Strain intensity, vorticity, and orientation of strain principal axes are easily obtained from the velocity field resulting from the streamfunction defined by Eq. (4). In particular, the orientation of strain principal axes is a piecewise function:

$$
\Phi=\left\{\begin{array}{l}
0 \quad \text { for } \sigma_{s}>0 \\
\pi / 2 \text { for } \sigma_{s}<0 .
\end{array}\right.
$$

The scalar gradient, G, thus undergoes an unsteady forcing through its orientation with respect to the strain principal axes; when it is aligned with the compressional direction, a sudden $\pi / 2$-step in $\Phi$ reverses this alignment by rushing the extensional axis against $G$. This special alignment dynamics was already analyzed for the passive vector [27].

The study was made for the pure unsteady flow $(\delta=0)$, the pure steady flow $(\delta=\sqrt{3 / 2})$, and a combination of both $(\delta=0.7)$. The velocity field is composed of alternating vortices and straining regions [23]; unless $\delta=\sqrt{3 / 2}$, this spatial structure is unsteady. In this simple flow, the Okubo-Weiss parameter, $Q=\sigma^{2}-\omega^{2}$, reliably distinguishes prevailing strain $(Q>0)$ from prevailing rotation $(Q<0)$. As $\Phi$ essentially takes constant values separated by sudden steps, a Lagrangian criterion including $D \Phi / D t[5]$ does not bring further information in the case under study.

\section{Numerical solution}

\subsection{Non-diffusive scalar}

When neglecting molecular diffusion, the Lagrangian dynamics of the scalar gradient orientation was derived from the non-diffusive form of Eq. (3),

$$
\frac{D \theta}{D t}=\frac{1}{2}\{\omega-\sigma \cos [2(\theta+\Phi)]\}
$$

together with the tracking of particles, solving $D \times / D t=\mathrm{u}$ with $\times$ being the position vector, and $\mathrm{u}$ the analytic velocity field. In Eq. (5), $\sigma, \omega$, and $\Phi$ were known analytically at each position of a particle. The numerical method was a fourthorder, explicit Runge-Kutta scheme. The Lagrangian particles were initially set on a $200 \times 200$ uniform mesh over the spatial domain $(0 \leq x \leq 2 \pi, 0 \leq y \leq 2 \pi)$, and Lagrangian statistics were achieved over the instantaneous values obtained along all the Lagrangian paths. The numerical procedure was run until the statistics essentially, the probability density functions (p.d.f's) - converged.

\subsection{Diffusive scalar}

The Lagrangian approach is less straightforward in the diffusive case. The numerical procedure included a Eulerian and a Lagrangian steps.

- Eulerian step: the Eulerian equation for G,

$$
\frac{\partial \mathrm{G}}{\partial t}+\mathrm{u} \cdot \nabla \mathrm{G}=-\mathrm{A}^{T} \cdot \mathrm{G}+D \Delta \mathrm{G}
$$


was solved at each time step for the components of $\mathrm{G}, G_{1}$ and $G_{2}$. The numerical domain was a $2 \pi \times 2 \pi$ square with periodic boundary conditions. The finite difference scheme was fourth-order in space [28], and the resolution was explicit in time. The gridding was uniform, with $\Delta x=\Delta y$.

The initial conditions for $\mathrm{G}$ were:

$$
G_{1}(x, y, 0)=0.5 \sin x \quad ; \quad G_{2}(x, y, 0)=1+0.5 \cos y .
$$

With these conditions, the mean scalar gradient has a non-zero component along the $y$ direction that keeps up the scalar gradient field;

- Lagrangian step: Equation (1) was solved for the components of G along the Lagrangian trajectories. As in the non-diffusive case, a fourth-order, explicit Runge-Kutta scheme was used. The components of $A^{T}$ were known analytically at each point.

At each particle position, the diffusive terms were computed with a five-point approximation. When computing the second-order spatial derivative in $x$ (resp. $y$ ) direction, the central point was given by the particle position. The values of $G_{1}$ and $G_{2}$ at the other four points in $x$ (resp. $y$ ) were interpolated over the Eulerian field.

Components $G_{1}$ and $G_{2}$ were subsequently used to derive the Lagrangian orientation and norm of vector $\mathrm{G}$.

The main parameter of the analysis is the Péclet number, $P e=U L / D$, where $U$ and $L$ are a velocity- and a lengthscale, respectively. In this study, $L=2 \pi$ and $U=\sqrt{3}$ - the value around which the maximum velocity within the field oscillates. All quantities are nondimensional.

In the diffusive case, two values of $P e$, namely 10 and 80 , were considered. The mesh size was $150 \times 150$ and $400 \times 400$, respectively.

\section{Results and discussion}

\subsection{Infinite Péclet number}

\subsubsection{Prevailing strain}

When strain prevails, and if strain persistence - the ratio of effective rotation to strain - is constant, the non-diffusive orientation equation [Eq. (6)] has a stable fixed point that defines an equilibrium orientation resulting from the competing actions of strain and rotation [5]. If strain persistence varies along Lagrangian trajectories, this direction expresses local equilibrium; it may be seen as the orientation the scalar gradient would take if its response was infinitely fast as compared to strain persistence variations. In the present case, the local equilibrium orientation within the strain eigenframe is given by $\zeta_{e q}=-\arccos (\omega / \sigma)-$ with $\sigma^{2}>\omega^{2}$; variable $\zeta$ is defined as $\zeta=2(\theta+\Phi)$. Note that $\zeta_{e q}$ is distinct from the compressional direction $\left(\zeta_{c}=-\pi / 2\right)$; it is only for $\sigma^{2} \gg \omega^{2}$ that $\zeta_{e q} \simeq \zeta_{c}$. The equilibrium direction lies within the 'compression area', for $\left|\theta_{e q}-\theta_{c}\right|=\pi / 4$ at most.

Figure 1 shows the p.d.f of $\left(\zeta_{c}-\zeta_{e q}\right) / \pi$ for $\delta=0,0.7$, and $\sqrt{3 / 2}$. The mean angular gap, $\left\langle\left(\zeta_{c}-\zeta_{e q}\right)\right\rangle / \pi$, between the compressional and equilibrium directions is 0.12 for $\delta=0$ and $\sqrt{3 / 2}$, but is larger $(0.18)$ for $\delta=0.7$. 

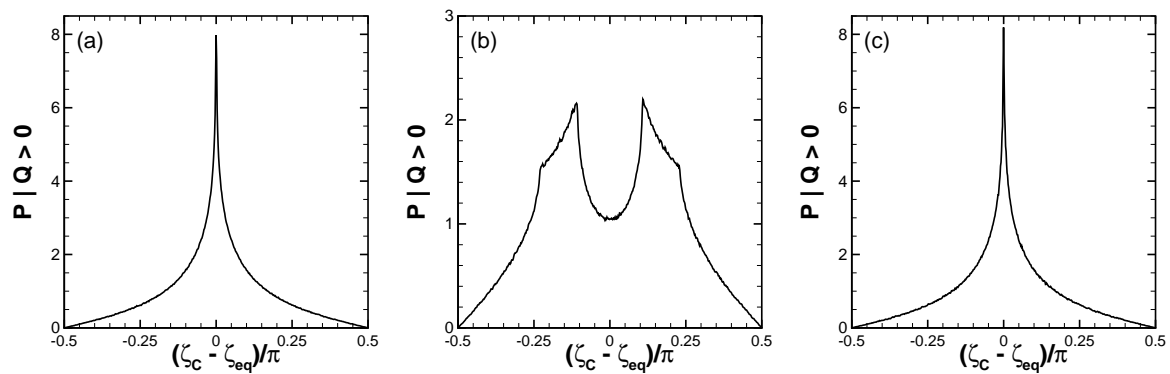

Fig. 1 P.d.f of angular gap between the compressional and the equilibrium directions, conditioned on prevailing strain; $\mathbf{a} \delta=0 ; \mathbf{b} \delta=0.7 ; \mathbf{c} \delta=\sqrt{3 / 2}$

Incidentally, Fig. 2 clearly shows that it is the equilibrium direction that draws the scalar gradient; the negative values of $S_{e q}=\operatorname{sign}\left(\theta-\theta_{e q}\right) . D \theta / D t$ are definitely more frequent than those of $S_{c}=\operatorname{sign}\left(\theta-\theta_{c}\right) \cdot D \theta / D t$, as measured by $A_{e q}^{-}=$ $\int_{-1}^{0} S_{e q} d S_{e q}$ and $A_{c}^{-}=\int_{-1}^{0} S_{c} d S_{c}$; as all Lagrangian angle derivatives, $D \theta / D t$ is normalized by $\left[0.5\left(\omega^{2}+\sigma^{2}\right)\right]^{1 / 2}$. Moreover, the negative values of $S_{c}$, in fact, result from the attraction of the scalar gradient toward the equilibrium direction when $\mathrm{G}$ is aligned outside the range $\left[\theta_{c}, \theta_{e q}\right]-$ or $\left[\theta_{e q}, \theta_{c}\right]$; when conditioning by $\theta$ being inside this range - i.e. $\left(\theta-\theta_{c}\right)\left(\theta-\theta_{e q}\right)<0-, S_{c}$ is essentially positive, which plainly shows that the attracting direction is not the compressional, but the equilibrium orientation.
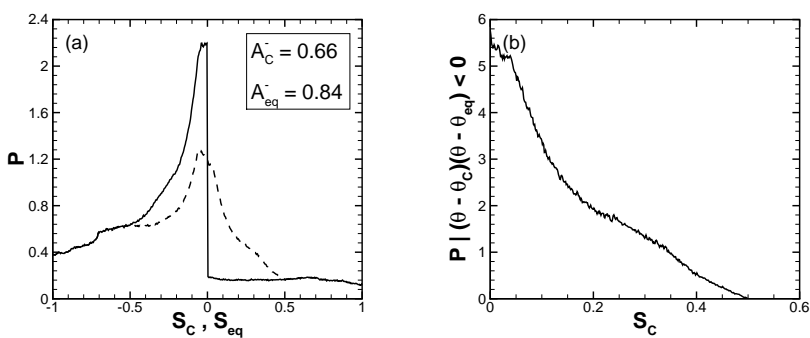

Fig. 2 a P.d.f's of $S_{e q}$ (solid line) and $S_{c}$ (dashed line); b P.d.f of $S_{c}$ conditioned on $(\theta-$ $\left.\theta_{c}\right)\left(\theta-\theta_{e q}\right)<0$; the p.d.f's are plotted for $\delta=0$ and prevailing strain

Alignment of the scalar gradient with the equilibrium direction indicates how far the orientation dynamics is adiabatic [12,13,29]. Adiabaticity of orientation dynamics has a physical ground. As suggested by alignment statistics, there is indeed some indication that the orientation dynamics of scalar gradient is adiabatic in turbulent flows. Preferential alignment with the equilibrium direction was attested in numerical simulations of two-dimensional turbulence [5] as well as 
in three-dimensional turbulence by using stochastic modelling [11]. Non-adiabatic regimes, on the other hand, may be achieved by monitoring non-turbulent flows, for example through any unsteady forcing device [29].

Consistently with the findings for the passive vector [27], the alignment dynamics is close to adiabaticity for $\delta=0.7$ and, to a lesser extent, for $\delta=0$. By contrast, it is non-adiabatic for $\delta=\sqrt{3 / 2}$. This feature is suggested by Fig. 3 displaying the p.d.f of $\zeta^{o}=\left(\zeta-\zeta_{\text {eq }}\right) / \pi$; note that $\left\langle\zeta^{\circ}\right\rangle=0.45,0.31$, and 0.65 for $\delta=0,0.7$, and $\sqrt{3 / 2}$, respectively. The degree of adiabaticity is even more clear from the p.d.f of the Lagrangian angle derivative plotted for $\delta=0,0.7$, and $\sqrt{3 / 2}$ (Fig. 4).
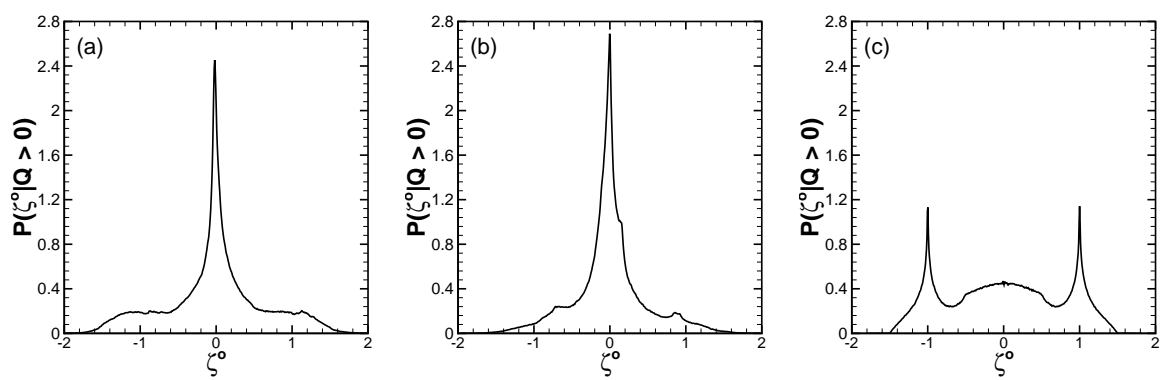

Fig. 3 P.d.f of angular gap between scalar gradient orientation and equilibrium direction, conditioned on prevailing strain; $\mathbf{a} \delta=0 ; \mathbf{b} \delta=0.7 ; \mathbf{c} \delta=\sqrt{3 / 2}$
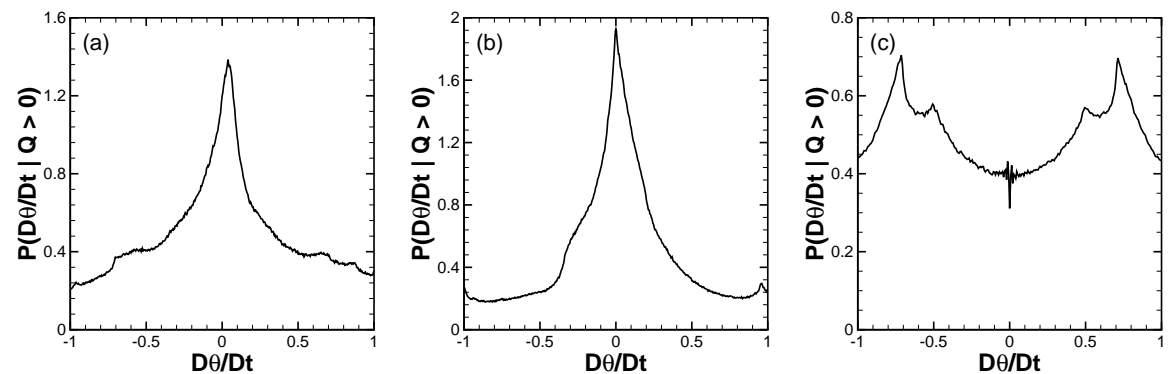

Fig. 4 P.d.f of the Lagrangian angle derivative, conditioned on prevailing strain; $\mathbf{a} \delta=0 ; \mathbf{b}$ $\delta=0.7 ; \mathbf{c} \delta=\sqrt{3 / 2}$

The p.d.f of the gap between $\zeta$ and the compressional direction, $\zeta^{\star}=(\zeta-$ $\left.\zeta_{c}\right) / \pi$, is plotted in Fig. 5. The scalar gradient experiences compressional strain within the range $-0.5 \leq \zeta^{\star} \leq 0.5$, and extensional strain outside. Interchanging the compressional strain and the extensional one, the scalar gradient dynamics is 
similar to the passive vector dynamics already analyzed in this model flow [27]. For $\delta=\sqrt{3 / 2}$, the scalar gradient spends half of the time under the influence of compression; the p.d.f peaks at $\zeta^{\star}=0$ and \pm 1 respectively correspond to alignment with compression and extension, and result from the bimodal regime caused by the alternating tilting of strain principal axes [27]. The double peak in the p.d.f of $\zeta^{\star}$ for $\delta=0.7$ is due to alignment with the equilibrium direction; the best alignment with the equilibrium direction occurs for $\delta=0.7$ (Fig. 3), and it is for the latter that the mean angular gap between the compressional and the equilibrium directions is the largest (Fig. 1).
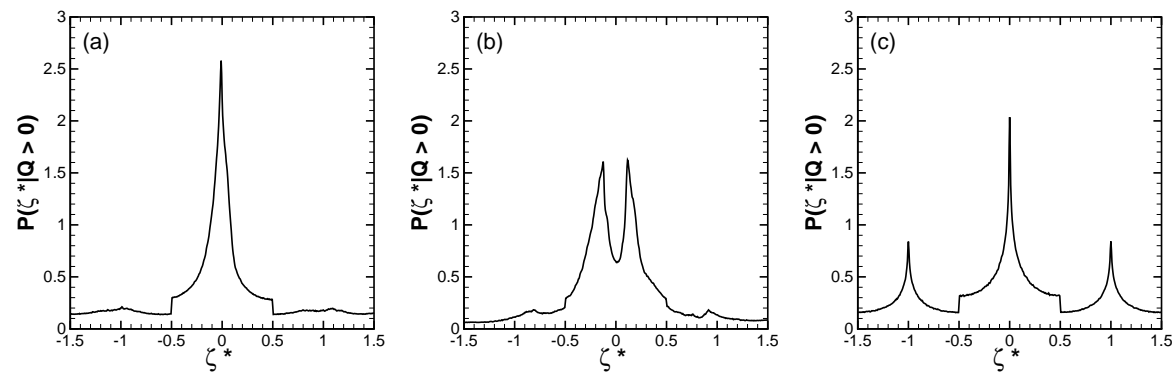

Fig. 5 P.d.f of angular gap between scalar gradient orientation and compressional direction, conditioned on prevailing strain; a $\delta=0 ; \mathbf{b} \delta=0.7 ; \mathbf{c} \delta=\sqrt{3 / 2}$

\subsubsection{Prevailing rotation}

As expected, the scalar gradient experiences a weaker influence of compression when rotation prevails, except in the case $\delta=\sqrt{3 / 2}$ for which the p.d.f of $\zeta^{\star}$ is, again, bimodal (Fig. 6).
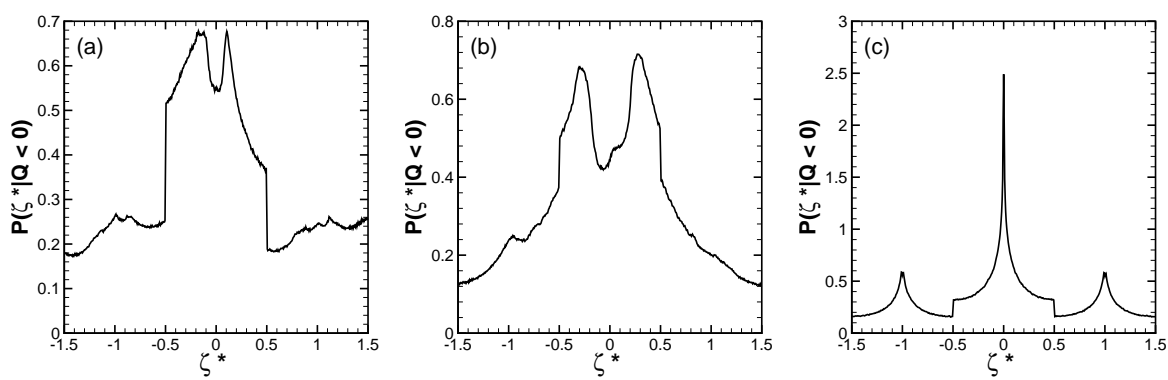

Fig. 6 P.d.f of angular gap between scalar gradient orientation and compressional direction, conditioned on prevailing rotation; $\mathbf{a} \delta=0 ; \mathbf{b} \delta=0.7 ; \mathbf{c} \delta=\sqrt{3 / 2}$ 
The orientation equation has no fixed point when rotation prevails [5], and the scalar gradient orientation never reaches equilibrium, as shown by the p.d.f of the Lagrangian angle derivative (Fig. 7). The p.d.f peaks near $D \theta / D t= \pm 0.7$ are easily explained by the fact that the normalized Lagrangian angle derivative, $(\omega-\sigma \cos \zeta) / 2 \cdot\left[\left(\omega^{2}+\sigma^{2}\right) / 2\right]^{-1 / 2}$, is close to $\pm \sqrt{2} / 2$ for $\omega^{2}>>\sigma^{2}$.
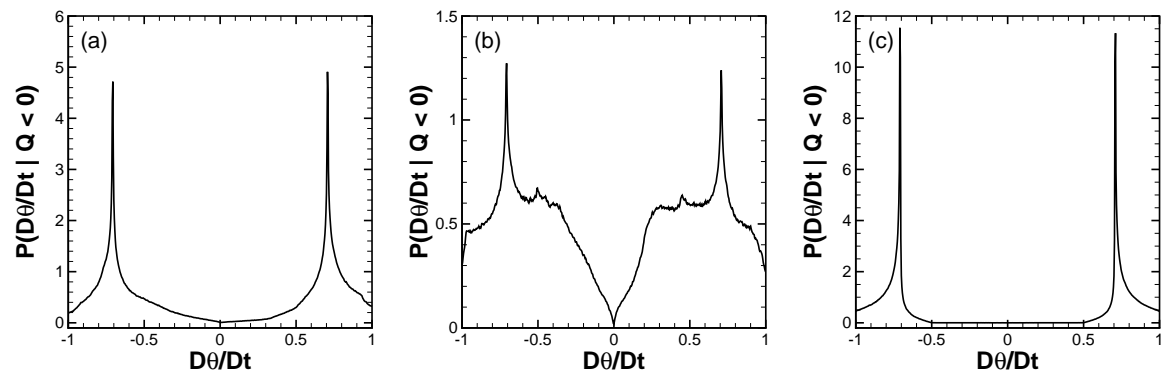

Fig. 7 P.d.f of the Lagrangian angle derivative, conditioned on prevailing rotation; a $\delta=0$; b $\delta=0.7 ; \mathbf{c} \delta=\sqrt{3 / 2}$

\subsection{Finite Péclet number}

\subsubsection{Prevailing strain}

In the diffusive case, $\theta_{e q}$ is not the fixed point of the orientation equation anymore, but is used as a reference direction to check the effect of molecular diffusion upon the scalar gradient orientation. Figure 8 displays the p.d.f's of $S_{e q}$, and $S_{m}=$ $\operatorname{sign}\left(\theta-\theta_{e q}\right) \cdot(D \theta / D t)_{m}$, for $\delta=0$, at $P e=80$ and $P e=10 ;(D \theta / D t)_{m}$ is the mechanical part of the Lagrangian derivative of orientation, as expressed by the first term of Eq. 3. The p.d.f of $S_{m}$ clearly shows the attraction of the equilibrium direction upon the scalar gradient; $A_{m}^{-}=\int_{-1}^{0} S_{m} d S_{m}=0.87$ for $P e=80$, and 0.98 for $P e=10$ - to be compared to 0.84 at infinite Péclet number (Fig. 3). The influence of molecular diffusion is accounted for by $S_{e q}$ - which is computed with the full Lagrangian derivative, $D \theta / D t$. As shown by the p.d.f of $S_{e q}$, the attraction exerted by the equilibrium direction is offset by molecular diffusion. However, it is still significant at $P e=10$ for which $A_{e q}^{-}=0.70\left(A_{e q}^{-}=0.75\right.$ at $\left.P e=80\right)$.

The p.d.f's of the angular gaps, $\zeta^{\circ}$ - with respect to the equilibrium direction - , and $\zeta^{\langle\rangle}$- with respect to the direction of the mean scalar gradient -, are plotted in Fig. 9, at $P e=80$ and 10 , for $\delta=0,0.7$, and $\sqrt{3 / 2}$. Except for $\delta=\sqrt{3 / 2}$, the p.d.f.'s of $\zeta^{\circ}$ at $P e=80$ are not much different from those computed at infinite Péclet number (Fig. 3).

If the scalar gradient dynamics is adiabatic (or at least nearly adiabatic) at infinite Péclet number $(\delta=0$ and $\delta=0.7)$, decreasing the latter makes the scalar gradient shifts away from the equilibrium direction as it is drawn towards the 

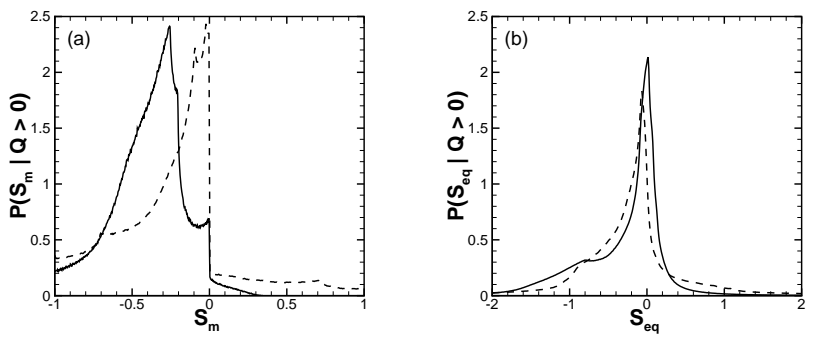

Fig. 8 a P.d.f of $S_{m}$; b p.d.f of $S_{e q} ; \delta=0$; dashed line: $P e=80$; solid line: $P e=10$

mean scalar gradient. This is especially clear for $\delta=0.7$; the p.d.f of $\zeta^{o}$ peaks at zero for $P e=80$ and then gets double-peaked, while the p.d.f of $\zeta^{\langle\rangle}$shows the opposite. This trend is confirmed at $P e=5$ (not shown). These results are consistent with the numerical simulations by Lapeyre et al. [19] who showed that molecular diffusion weakens alignment with the equilibrium direction.

In the non-adiabatic case $(\delta=\sqrt{3 / 2})$ the scenario is different. At infinite Péclet number, the alternating tilting of strain principal axes makes the scalar gradient align either with the compressional, or the extensional direction (Fig. 5). Decreasing the Péclet number alters these aligments by promoting alignment with the mean scalar gradient direction. Alignment with respect to the equilibrium direction gets even weaker as a result of misalignment with respect to the compressional direction. These results thus show molecular diffusion overcoming the mechanisms that rule the scalar gradient alignment at infinite Péclet number (Section 5.1.1), namely the competing actions of strain and rotation in the adiabatic regime, and the unsteady orientation of strain axes in the non-adiabatic one.

The p.d.f's of alignment with respect to the compressional direction are plotted in Fig. 10. They show a growing misalignment for the three values of $\delta$ as the Péclet number is decreased. For $\delta=0$ and $\delta=0.7$, the latter is of course caused by deviation from the equilibrium direction. The numerical simulations of threedimensional turbulence by Vedula et al. [21], too, show that misaligment with the compressional direction slightly increases when the Péclet number is decreased. One can also mention the simulations of Protas et al. [20] in two-dimensional turbulence, showing a growing misalignment of the vorticity gradient with decreasing Reynolds number.

The effect of molecular diffusion on the orientation dynamics is displayed by the p.d.f's of the Lagrangian angle derivatives, $D \theta / D t,(D \theta / D t)_{m}$, and $(D \theta / D t)_{d}$ - the diffusive part of $D \theta / D t$ - at $P e=10$ (Fig. 11). Note that $(D \theta / D t)_{m}$ is bounded, but that $(D \theta / D t)_{d}$ - and thus $D \theta / D t$ - are not; plots are however restricted to derivative values ranging within $[-1,1]$. Comparison with p.d.f's plotted at infinite Péclet number (Fig. 4) shows that molecular diffusion strongly alters the response of the scalar gradient to flow mechanical actions. For $\delta=0,(D \theta / D t)_{m}$ seriously departs from equilibrium; and this is even more obvious for $\delta=\sqrt{3 / 2}$. Equilibrium of $(D \theta / D t)_{m}$ is affected to a lesser extent for $\delta=0.7$, the value of $\delta$ ensuring quasi-adiabaticity of orientation dynamics at infinite Péclet number. The diffusive part of the Lagrangian derivative, $(D \theta / D t)_{d}$, is far from equilibrium 

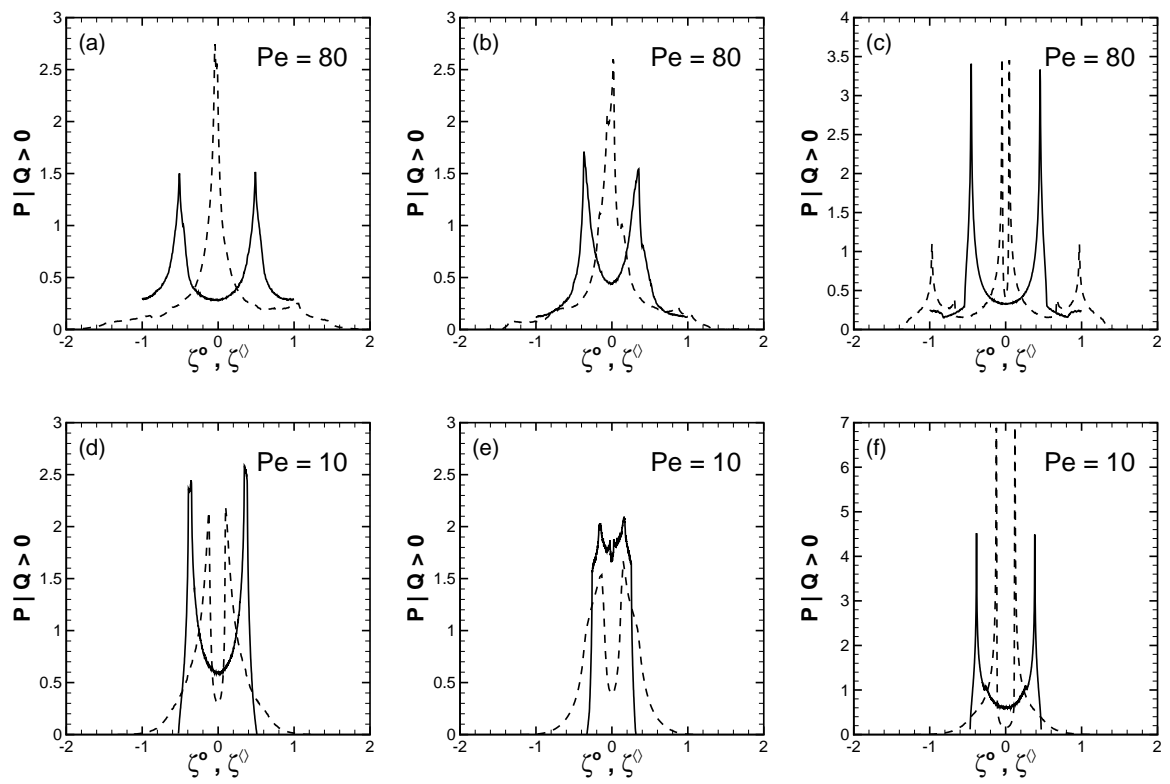

Fig. 9 P.d.f's of angular gaps, $\zeta^{o}$ (dashed line) and $\zeta^{\langle\rangle}$(solid line), for $P e=80$ and $P e=10$, conditioned on prevailing strain; $\mathbf{a}$ and $\mathbf{d} \delta=0 ; \mathbf{b}$ and $\mathbf{e} \delta=0.7 ; \mathbf{c}$ and $\mathbf{f} \delta=\sqrt{3 / 2}$

as well, no matter the value of $\delta$. However, the combination of $(D \theta / D t)_{m}$ and $(D \theta / D t)_{d}$ brings about equilibrium of the total derivative. The p.d.f of $D \theta / D t$ strongly peaks at zero for $\delta=\sqrt{3 / 2}$; the degree of equilibrium is lesser for $\delta=0$ and $\delta=0.7$.

\subsubsection{Prevailing rotation}

The effect of molecular diffusion on alignment p.d.f's is qualitatively similar when rotation prevails. The scenario is different, for at infinite Péclet number prevailing rotation precludes any equilibrium direction. As the Péclet number is decreased (Fig. 12), molecular diffusion tends to offset the rotation of the scalar gradient while promoting alignment with the direction of the mean scalar gradient. As a result, the moderate alignment with the compressional direction (Fig. 6) is weakened further.

Figure 13 shows that the mechanical part of the Lagrangian angle derivative is off equilibrium, as already observed at infinite Péclet number (Fig. 7). As in the case of prevailing strain, equilibrium of the total derivative is ensured by the combination of the mechanical and the diffusive parts. 

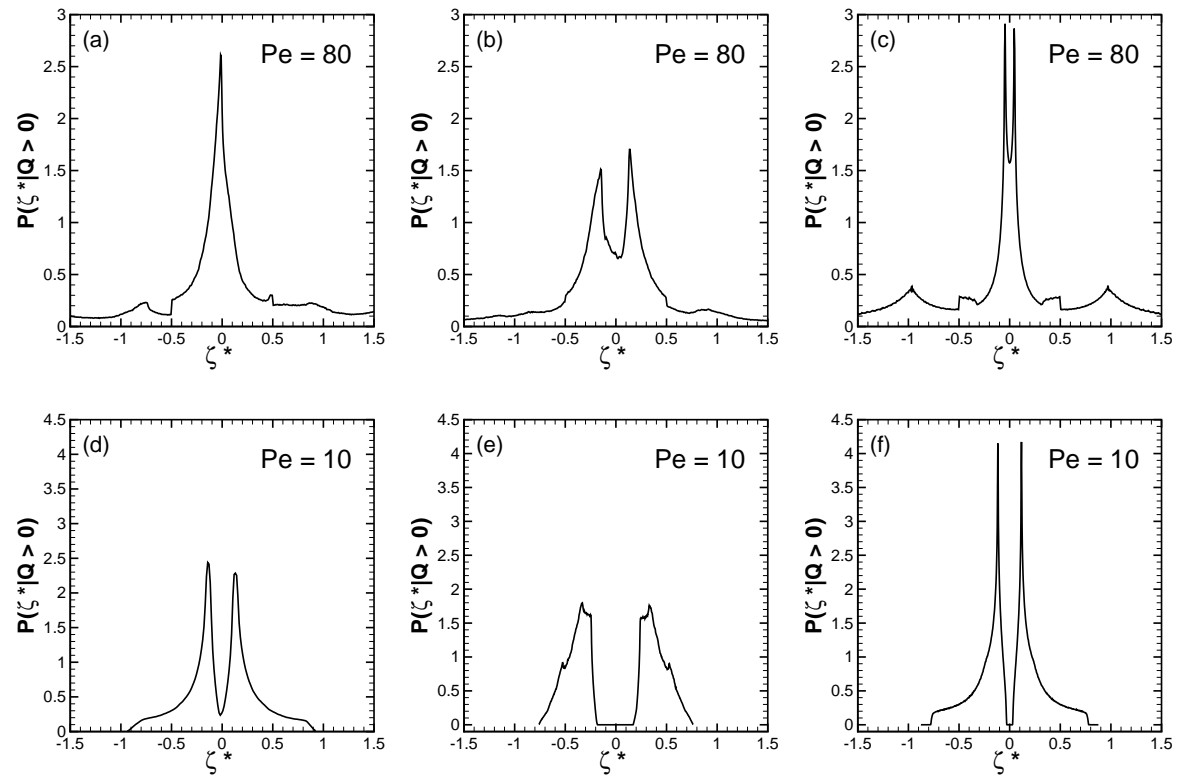

Fig. 10 P.d.f's of angular gap between scalar gradient orientation and compressional direction, for $P e=80$ and $P e=10$, conditioned on prevailing strain; $\mathbf{a}$ and $\mathbf{d} \delta=0 ; \mathbf{b}$ and $\mathbf{e} \delta=0.7 ; \mathbf{c}$ and $\mathbf{f} \delta=\sqrt{3 / 2}$
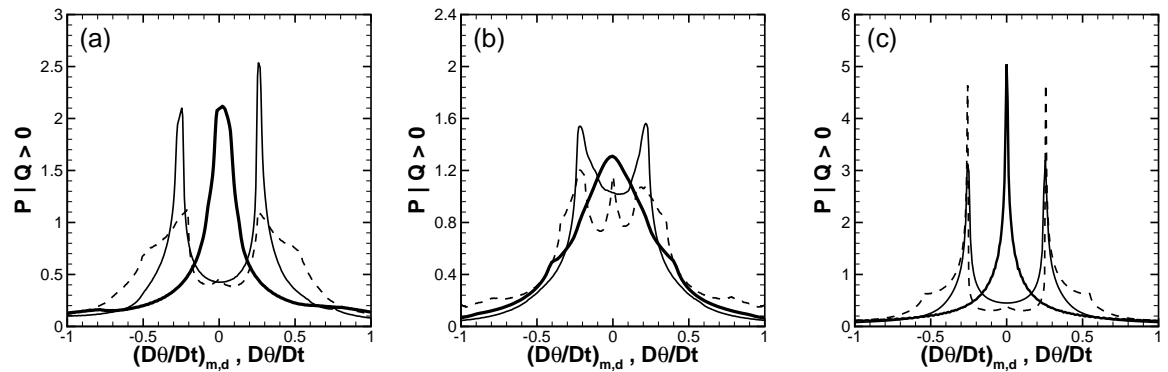

Fig. 11 P.d.f's of $(D \theta / D t)_{m}$ (dashed line), $(D \theta / D t)_{d}$ (thin solid line), and $D \theta / D t$ (bold solid line), for $P e=10$, conditioned on prevailing strain; a $\delta=0 ; \mathbf{b} \delta=0.7 ; \mathbf{c} \delta=\sqrt{3 / 2}$

\section{Conclusion}

The effect of molecular diffusion on the orientation dynamics of a scalar gradient was studied from a Lagrangian view in an analytic, parameterized, model flowfield. With regard to the degree of adiabaticity of the orientation dynamics at infinite Péclet number, three types of flow were considered: a steady flow $(\delta=\sqrt{3 / 2})$, an 

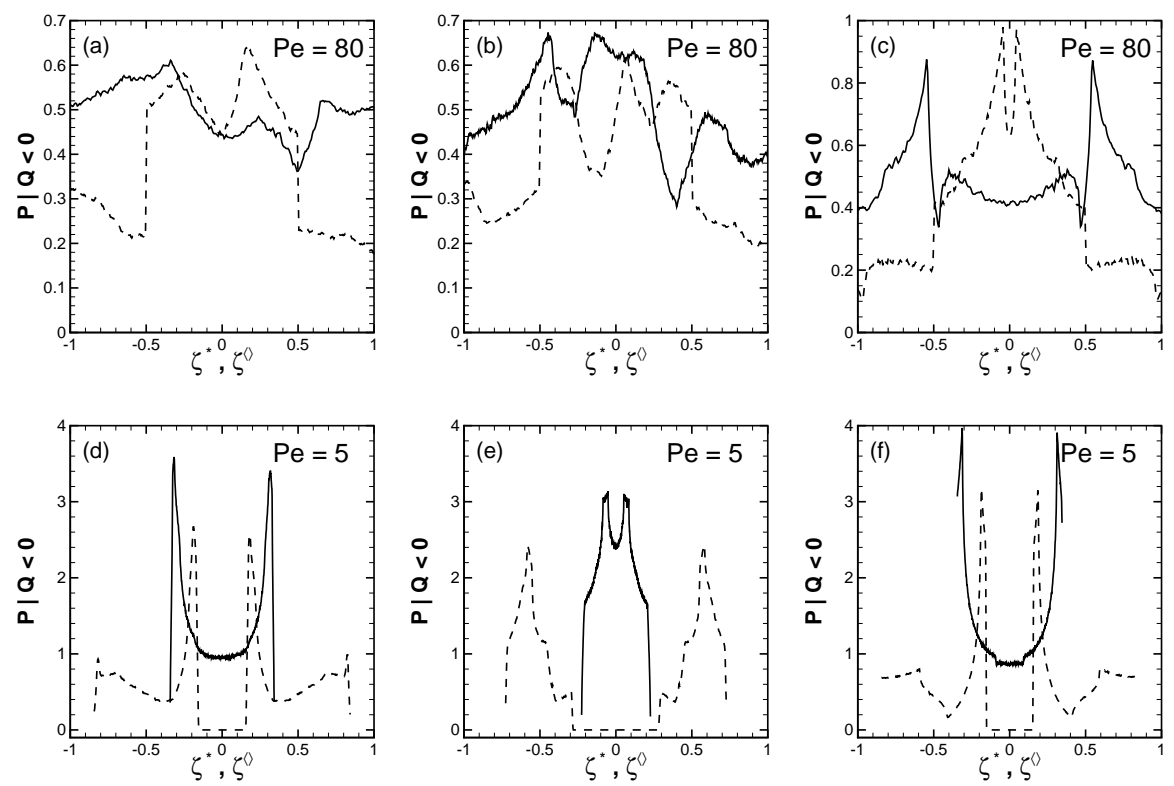

Fig. 12 P.d.f's of angular gaps, $\zeta^{*}$ (dashed line) and $\zeta^{\langle\rangle}$(solid line), for $P e=80$ and $P e=5$, conditioned on prevailing rotation; $\mathbf{a}$ and $\mathbf{d} \delta=0 ; \mathbf{b}$ and $\mathbf{e} \delta=0.7 ; \mathbf{c}$ and $\mathbf{f} \delta=\sqrt{3 / 2}$
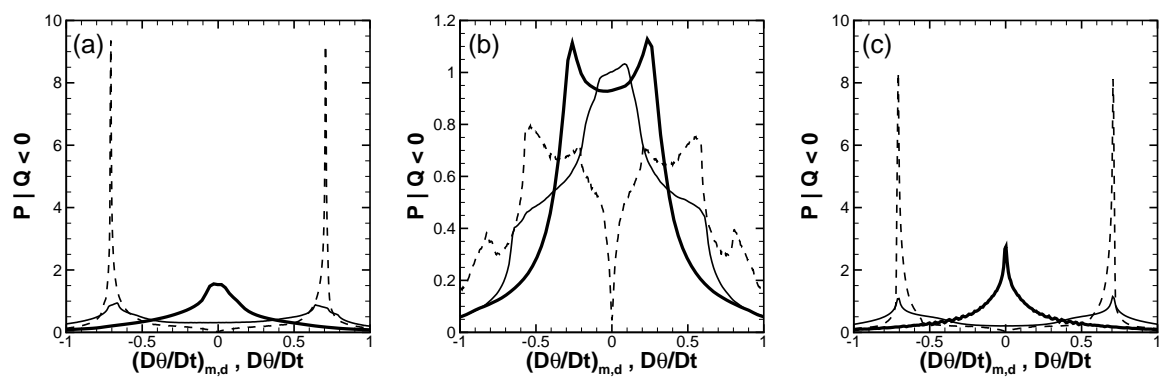

Fig. 13 P.d.f's of $(D \theta / D t)_{m}$ (dashed line), $(D \theta / D t)_{d}$ (thin solid line), and $D \theta / D t$ (bold solid line), for $P e=10$, conditioned on prevailing rotation; $\mathbf{a} \delta=0 ; \mathbf{b} \delta=0.7 ; \mathbf{c} \delta=\sqrt{3 / 2}$

unsteady flow $(\delta=0)$, and a combination of both $(\delta=0.7)$. From the former to the latter, adiabaticity of the orientation dynamics was increasing.

In the non-diffusive case, the numerical solution of the Lagrangian equation for the scalar gradient orientation was straightforward. When accounting for molecular diffusion, the Lagrangian orientation was derived from the Lagrangian equations for the scalar gradient components. Computing diffusive terms in these equations 
needed to simultaneously solve the Eulerian equations. Statistics were performed along the trajectories of Lagrangian particles. The scalar field was sustained by a mean gradient. Two values of the Péclet number were considered, namely $P e=80$ and $P e=10$.

At infinite Péclet number, Lagrangian statistics revealed the bare mechanical action of the flow upon the orientation dynamics of the scalar gradient:

- for prevailing strain, attraction of the scalar gradient towards the equilibrium direction - distinct from the compressional direction - is obvious. Orientation dynamics is close to equilibrium for $\delta=0$ and $\delta=0.7$ - near-adiabatic regime; it is off equilibrium for $\delta=\sqrt{3 / 2}$ - non-adiabatic regime. In the adiabatic case, the degree of alignment with the compressional direction is explained by the angular gap between the latter and the equilibrium direction;

- for prevailing rotation, orientation dynamics is off equilibrium no matter the value of $\delta$, and alignment with the compressional direction is poor, as expected.

These results were used as reference data to check the influence of molecular diffusion.

For finite Péclet number, the following results were derived:

- for prevailing strain, one has to distinguish whether the orientation dynamics at infinite Péclet number is adiabatic or not.

In the adiabatic case, the mechanical action of the flow still draws the scalar gradient towards the equilibrium direction, while molecular diffusion promotes alignment with the direction of the mean scalar gradient. At low Péclet number, the diffusive effect overcomes the mechanical action and draws the scalar gradient away from the equilibrium direction. As a result, the scalar gradient shifts away from the compressional direction as well. This scenario may take place in strain regions of turbulent flows.

In the non-adiabatic case, molecular diffusion tends to offset the effect of unsteadiness of strain principal axes orientation - that leads to a bimodal alignment of the scalar gradient in the present case. This kind of situation may occur in non-turbulent flows undergoing external unsteady forcing;

- for prevailing rotation, molecular diffusion competes with the unceasing rotation of the scalar gradient within the strain eigenframe, and promotes alignment with the direction of the mean scalar gradient; as a result, alignment with the compressional direction gets even weaker than it is at infinite Péclet number.

In this analytic model flowfield, the study clearly shows the influence of molecular diffusion on alignments as well as on the orientation dynamics resulting from the mechanical action of the flow. In particular, even at moderate Péclet number, the adiabatic response of the scalar field to the flow is deeply weakened. Misalignment with respect to the compressional direction is consistent with the findings of previous studies, but is precisely explained. Similar detailed Lagrangian statistics would deserve to be performed in more general flows, in particular, in isotropic and in forced turbulence.

\section{References}

1. Saffman, P.G.: On the fine-scale structure of vector fields convected by a turbulent fluid. J. Fluid Mech. 16, 545-572 (1963) 
2. Tsinober, A.: An Informal Conceptual Introduction to Turbulence. Springer, Dordrecht(2009)

3. Ashurst, Wm.T., Kerstein, A.R., Kerr, R.M., Gibson, C.H.: Alignment of vorticity and scalar gradient with strain rate in simulated Navier-Stokes turbulence. Phys. Fluids 30, 2343-2353 (1987)

4. Dresselhaus, E., Tabor, M.: The kinematics of stretching and alignment of material elements in general flow fields. J. Fluid Mech. 236, 415-444 (1992)

5. Lapeyre, G., Klein, P., Hua, B.L.: Does the tracer gradient align with strain eigenvectors in 2D turbulence? Phys. Fluids 11, 3729-3737 (1999)

6. Lüthi, B., Tsinober, A., Kinzelbach, W.: Lagrangian measurements of vorticity dynamics in turbulent flow. J. Fluid Mech. 528, 87-118 (2005)

7. Polygiannakis J.M., Moussas X.: On the role of strain and vorticity in plasmas. Plasma Phys. Control. Fusion 41, 967-983 (1999)

8. Favier, B., Bushby, P.J.: Small-scale dynamo action in rotating compressible convection. J. Fluid Mech. 690, 262-287 (2012)

9. Diamessis, P.J., Nomura, K.K.: Interaction of vorticity, rate-of-strain, and scalar gradient in stratified homogeneous sheared turbulence. Phys. Fluids 12, 1166-1188 (2000)

10. Garcia, A., Gonzalez, M.: Analysis of passive scalar gradient alignment in a simplified three-dimensional case. Phys. Fluids 18, 058101 (2006)

11. Gonzalez, M.: Kinematic properties of passive scalar gradient predicted by a stochastic Lagrangian model. Phys. Fluids 21, 055104 (2009)

12. Garcia, A., Gonzalez, M., Paranthoën, P.: On the alignment dynamics of a passive scalar gradient in a two-dimensional flow. Phys. Fluids 17, 117102 (2005)

13. Garcia, A., Gonzalez, M., Paranthoën, P.: Nonstationary aspects of passive scalar gradient behaviour. Eur. J. Mech. B 27, 433-443 (2008)

14. Jeong, E., Girimaji, S.S.: Velocity-gradient dynamics in turbulence: Effect of viscosity and forcing. Theor. Comput. Fluid Dyn. 16, 421-432 (2003)

15. Meneveau, C.: Lagrangian dynamics and models of the velocity gradient tensor in turbulent flows. Annu. Rev. Fluid Mech. 43, 219-245 (2011)

16. Holzner, M., Guala, M., Lüthi, B., Liberzon, A., Nikitin, N., Kinzelbach, W., Tsinober, A.: Viscous tilting and production of vorticity in homogeneous turbulence. Phys. Fluids 22, 061701 (2010)

17. Kida, S., Takaoka, M.: Breakdown of frozen motion of vorticity field and vorticity reconnection. J. Phys. Soc. Jpn. 60, 2184-2196 (1991)

18. Constantin, P., Procaccia, I., Segel, D.: Creation and dynamics of vortex tubes in threedimensional turbulence. Phys. Rev. E 51, 3207-3222 (1995)

19. Lapeyre, G., Hua, B.L., Klein, P.: Dynamics of the orientation of active and passive scalars in two-dimensional turbulence. Phys. Fluids 13, 251-264 (2001)

20. Protas, B., Babiano, A., Kevlahan, N.K.-R.: On geometrical alignment properties of twodimensional forced turbulence. Physica D 128, 169-179 (1999)

21. Vedula, P., Yeung, P.K., Fox, R.O.: Dynamics of scalar dissipation in isotropic turbulence: a numerical and modelling study. J. Fluid Mech. 433, 29-60 (2001)

22. Brandenburg, A., Procaccia, I., Segel, D.: The size and dynamics of magnetic flux structures in magnetohydrodynamic turbulence. Phys. Plasmas 2, 1148-1156 (1995)

23. Gonzalez, M.: Influence of molecular diffusion on alignment of vector fields: Eulerian analysis. Theor. Comput. Fluid Dyn. 31, 211-220 (2017)

24. Tanner, S.E.M., Hughes, D.W.: Fast-dynamo action for a family of parameterized flows. Astrophys. J. 586, 685-691 (2003)

25. Galloway, D.J., Proctor, M.R.E.: Numerical calculations of fast dynamos in smooth velocity fields with realistic diffusion. Nature 356, 691-693 (1992)

26. Childress, S., Soward, A.M.: Scalar transport and alpha-effect for a family of cat's-eye flows. J. Fluid Mech. 205, 99-133 (1989)

27. Gonzalez, M.: Effect of orientation dynamics on the growth of a passive vector in a family of model flows. Fluid Dyn. Res. 46, 015510 (2014)

28. Lele, S.K.: Compact finite difference schemes with spectral-like resolution. J. Comput. Phys. 103, 16-42 (1992)

29. Gonzalez, M., Paranthoën, P.: On the role of unsteady forcing of tracer gradient in local stirring. Eur. J. Mech. B 29, 143-152 (2010) 